\title{
Baltruschat, Astrid
}

\section{Die "Sache" des Unterrichts in der Unterrichtsforschung}

Zeitschrift für interpretative Schul- und Unterrichtsforschung 5 (2016) 1, S. 93-110

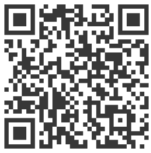

Quellenangabe/ Reference:

Baltruschat, Astrid: Die "Sache" des Unterrichts in der Unterrichtsforschung - In: Zeitschrift für

interpretative Schul- und Unterrichtsforschung 5 (2016) 1, S. 93-110 - URN:

urn:nbn:de:0111-pedocs-172014 - DOI: 10.25656/01:17201

https://nbn-resolving.org/urn:nbn:de:0111-pedocs-172014

https://doi.org/10.25656/01:17201

in Kooperation mit / in cooperation with:

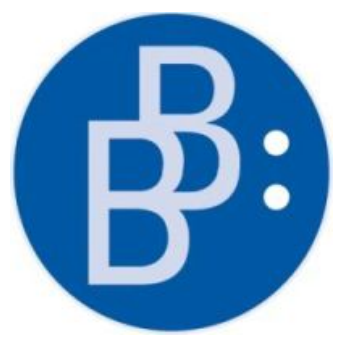

https://www.budrich.de

\section{Nutzungsbedingungen}

Gewährt wird ein nicht exklusives, nicht übertragbares, persönliches und beschränktes Recht auf Nutzung dieses Dokuments. Dieses Dokument ist ausschließlich für den persönlichen, nicht-kommerziellen Gebrauch bestimmt. Die Nutzung stellt keine Übertragung des Eigentumsrechts an diesem Dokument dar und gilt vorbehaltlich der folgenden Einschränkungen: Auf sämtlichen Kopien dieses Dokuments müssen alle Urheberrechtshinweise und sonstigen Hinweise auf gesetzlichen Schutz beibehalten werden. Sie dürfen dieses Dokument nicht in irgendeiner Weise abändern, noch dürfen Sie dieses Dokument für öffentliche oder kommerzielle Zwecke vervielfältigen, öffentlich ausstellen, aufführen, vertreiben oder anderweitig nutzen.

Mit der Verwendung dieses Dokuments erkennen Sie die Nutzungsbedingungen an.

\section{Terms of use}

We grant a non-exclusive, non-transferable, individual and limited right to using this document.

This document is solely intended for your personal, non-commercial use. Use of this document does not include any transfer of property rights and it is conditional to the following limitations: All of the copies of this documents must retain all copyright information and other information regarding legal protection. You are not allowed to alter this document in any way, to copy it for public or commercial purposes, to exhibit the document in public, to perform, distribute or otherwise use the document in public.

By using this particular document, you accept the above-stated conditions of use.

\section{Kontakt / Contact:}

\section{peDOCS}

DIPF | Leibniz-Institut für Bildungsforschung und Bildungsinformation Informationszentrum (IZ) Bildung

E-Mail: pedocs@dipf.de

Internet: www.pedocs.de

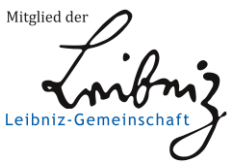




\section{ZISU}

\section{Zeitschrift für interpretative Schul- und Unterrichtsforschung}

\section{Editorial}

Hedda Bennewitz,

Ergebniskulturen

Bernd Hackl,

Torsten Pflugmacher

\section{Thementeil}

Christoph Leser

Jochen Heins

Maria Hallitzky,

Beate Beyer,

Christopher Hempel,

Christian Herfter,

Johanna Leicht,

Ellen Schroeter

Tanja Sturm
Vermitteln und Bewerten im Unterricht

Die Rolle von (Teil-)Ergebnissen im Aufgabenverstehens- und Textverstehensprozess zu einem literarischen Text

„Was muss'n jetzt nochmal auf den Strich?“

Zur Konstitution von Ergebnisräumen im

Literaturunterricht der Sekundarstufe

\section{Allgemeiner Teil}

\begin{tabular}{|c|c|}
\hline Andreas Gruschka & Was heißt „bildender Unterricht“? \\
\hline Astrid Baltruschat & Die Sache des Unterrichts in der Unterrichtsforschung \\
\hline $\begin{array}{l}\text { Christoph Bräuer, } \\
\text { Kerstin Rabenstein, } \\
\text { Svenja Strauß }\end{array}$ & $\begin{array}{l}\text { Wie findet Literaturunterricht mit textproduktiven } \\
\text { Verfahren seine Form? Eine explorative Studie am } \\
\text { Beispiel von Herrndorfs „Tschick“ }\end{array}$ \\
\hline Uwe Hericks & $\begin{array}{l}\text { „Es sollte am Schluss ein deutscher Satz } \\
\text { rauskommen, nicht?“ - Rekonstruktionen } \\
\text { zur Entstehung mathematischen Wissens im } \\
\text { Schulunterricht }\end{array}$ \\
\hline
\end{tabular}


Alexa Hempel, Detlef Pech

\section{Rezensionen}

Markus Hoffmann
Kinder erforschen Geschichte -

148
Zeitzeug/-inneninterviews zur deutschen Teilung

Bräu, Karin/Schlickum, Christine (Hrsg.) (2015):

Soziale Konstruktionen in Schule und Unterricht.

Zu den Kategorien Leistung, Migration, Geschlecht,

Behinderung, Soziale Herkunft und deren

Interdependenzen. Opladen, Berlin, Toronto: Budrich.

ISBN 978-3-8474-0689-1. 


\section{Die „Sache“ des Unterrichts in der Unterrichtsforschung}

\section{Zusammenfassung}

Empirische Forschung ist prinzipiell auf klare Begriffe angewiesen, um die beforschte Wirklichkeit präzise erfassen zu können. Trotz vielfältiger Bemühungen um theoretische Modellierungen, die es ermöglichen, das Spezifische des Unterrichts empirisch zu erfassen, gibt es in der qualitativen Unterrichtsforschung einen breiten Konsens darüber, dass diese noch nicht zu einem befriedigenden Ergebnis geführt haben. Eine Klärung des Begriffs des Unterrichtsgegenstands, also der „Sache“, um die es im Unterricht geht, könnte einen wesentlichen Beitrag zur Behebung dieses Desiderats leisten. Im Zentrum dieses Beitrags stehen die Präzisierung dieses Begriffs und seine Verortung in bereits bewährten theoretischen Entwürfen von Prange und Sünkel. Nachdem die Annäherung an den Begriff des Unterrichtsgegenstands zunächst über eine empirische Rekonstruktion erfolgt, wird er schließlich seinerseits als Kategorie für die empirische Analyse erprobt.

Schlagwörter: Unterrichtsforschung; Unterrichtstheorie; Empirie-Theorie; Unterrichtsgegenstand

\section{The subject matter of teaching and learning in classroom research}

Empirical research is in principle dependent on clear terms to accurately capture the explored reality. Despite a variety of efforts to theoretical modeling, that allow to capture the specific of teaching and learning empirically, there is a broad consensus in qualitative classroom research, that this has not yet achieved a satisfactory result. A clarification of the concept of the subject matter, i.e. the "cause" in the classroom, could be a significant contribution to resolve this Desideratum. In the center of this article is the clarification of this concept and its positioning in already proven theoretical designs by Prange and Sünkel. After the alignment with the concept of the object of lesson first on an empirical reconstruction, it will finally tested as a category in the empirical analysis.

Keywords: classroom research; theory of teaching and learning; empiric approach - theoretical approach; subject matter of teaching and learning

Bei der Auseinandersetzung mit unterschiedlichen qualitativ-empirischen Interpretationen ein und derselben Unterrichtssequenz kam die Frage nach dem Stellenwert der Sache des Unterrichts in den Blick. Sie wurde zum Anlass, diesem Aspekt im folgenden Beitrag systematisch nachzugehen und seine Bedeutung für die Unterrichtsforschung auszuloten.

Damit wird ein Diskurs berührt, der in den letzten Jahren in der deutschsprachigen qualitativ-empirischen Unterrichtsforschung zunehmend an Bedeutung gewonnen hat, nämlich die Frage danach, was das Spezifische des Unterrichts sei und in welcher Weise dies in der empirischen Unterrichtsforschung zu berücksichtigen wäre (Meseth/Proske/ Radtke 2011; Combe 2013; Hünig/Kabel 2014; Geier/Pollmanns 2016). Diese Fragestellung spielt international bislang (noch) keine Rolle. Dass sie hierzulande auftaucht, hängt sicherlich nicht zuletzt mit dem Nährboden der German Tradition der Didaktik“ (Arnold 2009: 17; vgl. Hudson/Meyer 2011: 16; Shirley 2008) und der damit einher- 
gehenden Sensibilität für genuin pädagogische und didaktische Begrifflichkeiten und Konzepte zusammen. ${ }^{1}$

\section{Empirische Annäherung an ein theoretisches Problem}

Ausgangspunkt des Beitrags ist die videografisch aufgezeichnete Unterrichtssequenz aus einer Mathematikstunde einer 8. Klasse Realschule aus dem Fallarchiv HILDE der Universität Hildesheim (Archiv Nr. 18)². Ein und derselbe Fall sollte aus verschiedenartigen Perspektiven beleuchtet und diese ihrerseits reflektiert werden, um das Potenzial einer fallorientierten Rekonstruktionsforschung auszuloten (Pieper 2014: 12). Vier Wissenschaftlerinnen waren beteiligt: So interpretierte Fredrike Kern (2014) diese Sequenz aus gesprächsanalytischer Sicht, Iris Nentwig-Gesemann (2014) nutzte dafür die Dokumentarische Methode und Babara Schmidt-Thieme (2014) betrachtete sie aus mathematikdidaktischer Perspektive. Bei der sich anschließenden Metaanalyse der Interpretationen von Baltruschat (2014) kam zum Vorschein, dass in allen Primäranalysen der Begriff der Interaktion wie selbstverständlich gebraucht, jedoch sehr unterschiedlich konzeptioniert wurde (ebd.: 156f.). Während die beiden sozialwissenschaftlichen Vertreterinnen im Kontext von Gesprächsanalyse und Dokumentarischer Methode mit einem Interaktionsbegriff in der Tradition Georg Herbert Meads (1968) operierten und die Wortbeiträge von Lehrerin und Schüler/-innen als Akte und Re-Akte direkt aufeinander bezogen, kam in der fachdidaktischen Interpretation eine weitere Instanz ins

1 Diese Fragestellung beschäftigt hierzulande ausschließlich qualitativ-empirisch forschende Wissenschaftlerinnen und Wissenschaftler, obwohl auch Vertreterinnen und Vertreter der quantitativ-empirischen Richtung eingeladen waren, sich an diesem Diskurs zu beteiligen (Meseth/Proske/Radtke 2011: 7). Warum es von ihrer Seite zu dieser Problemstellung bislang noch keine eigenen Beiträge gibt, liegt vermutlich an dem anders gearteten Verständnis von Forschung, ihrer disziplinären Orientierung an der Psychologie und in der anderen Verhältnisbestimmung zwischen Theorie und Empirie. Es würde den Rahmen dieses Beitrags sprengen, dies näher auszuführen. Nachdem international die quantitativ-empirische Forschung angelsächsischer Tradition eine vorherrschende Rolle spielt, überrascht es also nicht, dass dieser Fragestellung auch international bislang keine Bedeutung beigemessen wurde. Dass sie von Seiten der sinnverstehenden qualitativrekonstruktiven Forschung in diesem Rahmen noch nicht thematisiert wurde, liegt vermutlich nicht nur an der marginaleren Rolle, die diese dort spielt, sondern wohl auch an der geringen Bedeutung, die gegenstandstheoretischen Vorannahmen bislang beigemessen wurde. Auch wenn sich dies allmählich zu ändern scheint, wie man beispielsweise an der Erweiterung der 4. Auflage des Handbuchs „Doing Qualitative Research“ (Silverman 2013: 102-119) hinsichtlich dieses Aspekts ablesen kann, so müssten entsprechende begrifflich-theoretische Vorarbeiten, wie sie die German Tradition bietet, dort erst noch geleistet werden.

2 Das Fallarchiv HILDE (s.o.) enthält videografische Unterrichtsaufzeichnungen hauptsächlich aus den Fächern Deutsch, Mathematik, Sachunterricht und Sport, überwiegend aus den Jahrgangsstufen 3 bis 8 . Neben den Videoaufzeichnungen enthält das Archiv auch zugehörige Transkripte und weitere Dokumente wie Unterrichtsverlaufspläne, Lehrerreflexionen und Schülerrückmeldungen (vgl. Schmidt-Thieme 2014: 139). Vgl. auch: www.uni-hildesheim.de/celeb/forschung-und-wissenschaftlicher-nachwuchs/fallarchive/videobasiertes-fallarchiv-hilde/(abgerufen: 7.12.2015) 
Spiel, auf die hin, bzw. von der her die Aktionen der Beteiligten gedeutet und dementsprechend kategorisiert wurden. So wurde beispielsweise die Frage der Lehrerin an die Klasse „Wer könnte mal bitte dieses Parallelogramm benennen?“ als „Konstituierung der Aufgabe“ interpretiert und ein nachfolgender Wortwechsel zwischen Lehrerin und Schüler/-innen als „Fixierung der Lösung“ (Schmidt-Thieme 2014: 141). In den Bezeichnungen „Aufgabe“ und „Lösung“ spiegelt sich die implizite Bezugnahme auf den Unterrichtsgegenstand dieser Stunde wider. Er fungiert hier stillschweigend als eine Art Angelpunkt (vgl. Baltruschat 2014: 157). Einzig in der fachdidaktischen Interpretation wurden die Visualisierungen an Tafel und OHP als eigene Sinnstrukturen aufgenommen und als solche in die Analyse einbezogen. So wurde die projizierte Grafik (s. Abb. 1) als „Datum“ betrachtet und die Äußerungen der Schüler/-innen (z.B. Ich würde mal vermuten, dass alle dieselbe Fläche haben) als „Konklusionen“ darauf bezogen (SchmidtThieme 2014: 143f.).

Abbildung 1: Screenshot des Videos zur Unterrichtssequenz „Parallelogramme“

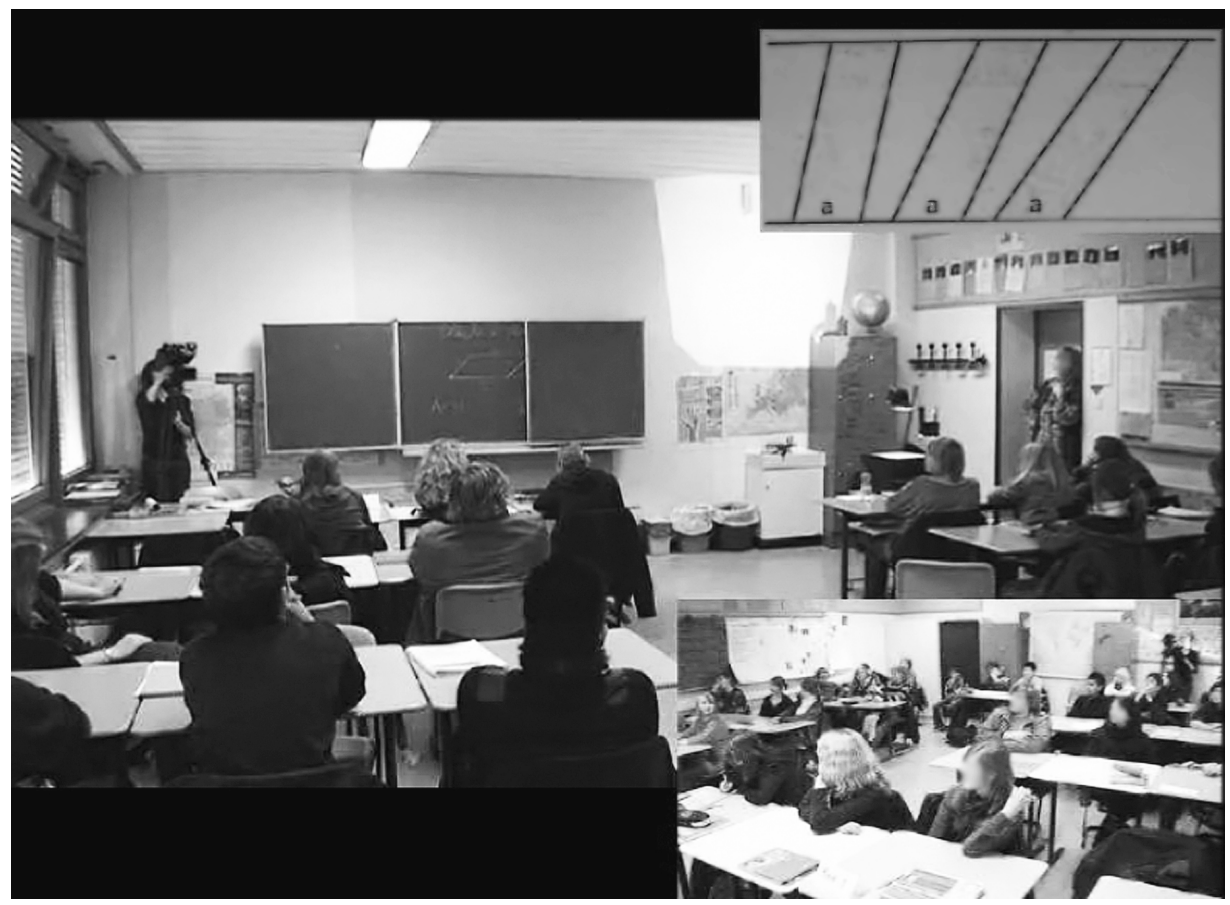

Betrachtet man diese Sequenz im Video, so fällt auf, dass sich die Lehrerin an den äußersten Rand des Klassenzimmers, in dessen Türöffnung, begeben hatte und die Blickschneise der Schüler auf die Projektion freigab (vgl. Abb. 1). Bei der Interaktion zwischen Lehrerin und Schüler/-innen sind die Blicke der Schüler/-innen meist auf die Projektion gerichtet, während die Blickrichtung der Lehrerin fast durchgängig auf die Klasse zielt und nur hin und wieder zur Projektion wandert. Die Lehrerin hat die Inter- 
aktion zwischen Schülern und Grafik herbeigeführt, indem sie die Folie auflegte und beschränkt sich nun darauf, die Schüler aufzurufen und mit $m h m \mathrm{zu}$,antworten'. Diese beziehen sich in ihren Äußerungen nicht primär auf die Lehrerin, sondern auf die Grafik und äußern ihre Vermutungen darüber, was dort abgebildet sei und welche geometrischen Zusammenhänge sie identifizieren können.

Den Schüler/-innen ist diese Praxis offensichtlich sehr vertraut, da sie ohne weitere Erläuterung darauf einsteigen. Es handelt sich hier offenbar um ein von Lehrerin und Schüler/-innen gemeinsam geteiltes handlungsleitendes Wissen darüber, ,wie Unterricht, bzw. Unterrichtsinteraktion ,funktioniert““ (vgl. Nentwig-Gesemann 2014: 133).

Während die sozialwissenschaftlichen Interpretinnen dieser ,Interaktion“ zwischen Schüler/-innen und Grafik keine weitere Aufmerksamkeit schenken und alle Akte und Re-Akte rein personal deuten, verzichtet die fachdidaktische Interpretin auf die personale Dimension dieser ,Interaktion‘. Es stellt sich die Frage, welcher Begriff von Interaktion geeigneter ist für die Beschreibung von Unterricht?

\section{Die Sache des Unterrichts als das Spezifische des Unterrichts}

In den letzten Jahren ist insbesondere in der qualitativ-empirischen Unterrichtsforschung um eine theoretische Modellierung gerungen worden, die sich als Basis für eine genuin erziehungswissenschaftliche bzw. pädagogische Unterrichtsforschung eignet (Meseth/ Proske/Radtke 2011; Combe 2013; Hünig/Kabel 2014; Geier/Pollmanns 2016). Immer wieder wurde Unbehagen darüber geäußert, dass das Spezifische des Unterrichts in den sozialwissenschaftlichen Ansätzen, auf die vor allem in der qualitativen Forschung zurückgegriffen wird, zu kurz käme - ohne jedoch eine Einigung darüber herbeiführen zu können, was denn nun genau das Spezifische des Unterrichts sei (Gruschka/Herzog/ Meseth/Proske/Reh 2011; Hünig/Kabel 2014).

In der qualitativ-empirischen Unterrichtsforschung kann man diesbezüglich drei unterschiedliche Strömungen ausmachen. Da sind zunächst diejenigen, die überzeugt sind, dass sich mittels der von ihnen vertretenen sozialwissenschaftlichen Ansätze auch das Spezifikum des Unterrichts abbilden ließe, da die Unterrichtssituation eben zu allererst eine soziale Situation sei. Hier könnte man die ethnografische Unterrichtsforschung (z.B. Breidenstein 2008) oder gesprächsanalytische Formen der Unterrichtsforschung (z.B. Kern 2014; Lüders 2011) anführen. In deutlicher Opposition dagegen fordert eine bildungstheoretisch orientierte Unterrichtsforschung, die einheimischen pädagogischen Begriffe wie Bildung, Erziehung und Didaktik als leitende Orientierungen in der empirischen Analyse zur Geltung zu bringen (Gruschka 2013). Die dritte Strömung könnte man als eine vermittelnde Position zwischen den beiden erstgenannten Ansätzen verstehen. Hier wird der Versuch gemacht, sozialwissenschaftliche Ansätze mit pädagogischen Konzepten zu verbinden, um die pädagogische Ordnung (Reh/Rabenstein/Idel 2011), die „Pädagogizität“ (Meseth/Proske/Radtke 2011b: 224) oder die Bildungswirksamkeit (Bonnet 2011) der sozialen Situation Unterricht erfassen zu können. Am weitesten verbreitet erscheint hier der Ansatz der Lernkulturanalyse von Reh, Rabenstein und Idel, 
die eine praxistheoretische Perspektive (Schatzki 1996, 2002) mit dem Konzept des Zeigens als pädagogischer Grundoperation (Prange 2005) verbinden.

Weniger eingebunden in diese Diskussion sind qualitativ forschende Fachdidaktiker/-innen in der Tradition des Mathematikdidaktikers Götz Krummheuer. Auf ihn geht der Begriff der ,Interaktion' zurück, den die fachdidaktische Interpretin im Eingangsbeispiel verwendete. Betrachtet man seinen Ansatz der Interaktionsanalyse, so erkennt man am verwendeten Begriffsinstrumentarium schnell, dass bereits bei ihm alle Interaktionen von der Sache des Unterrichts her gedacht und interpretiert werden. So werden Schüler/-innen beispielsweise als „Kreatoren“, „Paraphrasierer“ oder „Imitatoren“ bezeichnet (Krummheuer 2007), je nachdem, wie sich ihre Äußerungen zum Unterrichtsgegenstand der jeweiligen Lehr-Lern-Situation verhalten. Dieser Instanz, die hier als impliziter Bezugspunkt fungiert, soll im Folgenden näher nachgegangen werden.

Bereits die weit verbreitete basale Modellierung des sog. „didaktischen Dreiecks“ (Hudson/Meyer 2011: 18f.; Prange 2005: 55; Sünkel 2002: 63ff.) weist auf die fundamentale Bedeutung des Dritten oder der Sache des Unterrichts hin, die den grundlegenden Unterschied zu den zweipolig angelegten interaktionistischen Modellen markiert. In der Didaktik hat sich für dieses Dritte auch der Begriff des Unterrichtsgegenstands eingebürgert. Es lohnt sich, diesem Begriff etwas genauer nachzugehen. Anders als das Wort Unterrichtsgegenstand nahelegt, bezeichnet dieser Begriff nämlich niemals einen materiellen Gegenstand. Glöckel erläutert dies in Zusammenhang einer Reflexion des didaktischen Ideals der Gegenstandsnähe von Unterrichtsmedien:

Man könnte noch überlegen, ob der vorhandene reale Gegenstand selbst als „Medium“ des Unterrichts bezeichnet werden könne. Zunächst scheint die Antwort „nein“ zu lauten, weil etwas, das selbst da ist, doch keines Mittlers bedarf. So einfach ist die Sache aber nicht. Das reale vorgeschichtliche Steinbeil, der lebendige Feuersalamander, selbst der Bäckermeister Huber in seiner Backstube, sie alle sind ja gar nicht als Individuen gefragt; sie stehen als Beispiele für Steinbeile oder vorgeschichtliche Funde allgemein, für die Spezies der Feuersalamander oder die Klasse der Lurche, für die Arbeit des Bäckers oder handwerkliche Berufe überhaupt. In diesem Sinne sind sie doch Vertreter, Mittler eines allgemeineren Sachverhalts, um den es eigentlich geht. (...) Der zu lernende Sachverhalt ist im Grunde immer ein ideeller (Glöckel 2003: 42f.).

Der Hinweis auf die prinzipielle Immaterialität des Unterrichtsgegenstandes ist besonders für die empirische Unterrichtsforschung von Bedeutung, weil sie sich aufgrund ihres Vorgehens eben bevorzugt dem empirisch Erfassbaren, also dem Sichtbaren oder Hörbaren, zuwendet.

Wenn man nun die Sache des Unterrichts explizit als bedeutsame Kategorie in den Blick nehmen will, benötigt man einen präzisen Begriff. Andernfalls wird man geneigt sein, alle möglichen materiellen Dinge, die im Unterrichtsgeschehen vorkommen, als Unterrichtsgegenstand zu bezeichnen und damit eben genau den entscheidenden Bezugspunkt verfehlen.

Das bedeutet nicht, dass man die Dinge, die im Unterricht vorkommen, nicht einfach auch unvoreingenommen als Dinge betrachten und die Auseinandersetzung der Menschen mit diesen Dingen in den Fokus rücken könnte, so wie das in den letzten Jahren auch in der Unterrichtsforschung zunehmend getan wird (Nohl/Wulf 2013; Dörpinghaus/Nießeler 2012). Wenn man allerdings auf das Spezifische des Unterrichts 
fokussieren will und dabei zu dem Schluss kommt, dass die Kategorie des Unterrichtsgegenstandes dafür relevant ist, dann müsste man auch klären, in welchem Bezug die Dinge zu ihm stehen. Sie bekämen auch von dort her - und nicht nur von Zuschreibungen und Praktiken der Akteur/-innen - eine Bedeutung, aus der sich eine Systematik der Dinge im Unterricht ableitet. Bislang kommen in der Unterrichtsforschung eher vorreflexive rudimentäre Formen einer derartigen Systematik zum Tragen, beispielsweise wenn Unterrichtsmaterialien allgemein als Medien bezeichnet werden. Konsequent vom Unterrichtsgegenstand aus betrachtet, würde man dagegen die Bezeichnung Unterrichtsmedium nur für jene Dinge reservieren, die tatsächlich in der aktuellen Lehr-LernSituation auf diesen verweisen. Andere Dinge könnte man dann beispielsweise unter dem Begriff Unterrichtsaccessoire zusammenfassen und so verdeutlichen, dass ihnen eine andere Funktion im Unterrichtsgeschehen zukommt (vgl. Baltruschat 2015: 287f.). Etwas anders akzentuiert, aber ebenfalls vom Unterrichtsgegenstand her gedacht, ist die Einteilung in Medien und Hilfsmittel, die Glöckel vorschlägt (vgl. Glöckel 2003: 41).

\section{Der Unterrichtsgegenstand und das Zeigen}

Der systematische Entwurf Klaus Pranges, in dem er das Zeigen als die pädagogische Grundstruktur schlechthin ausweist (Prange 2005), ist in der qualitativen Forschung intensiv rezipiert worden (Reh/Ricken 2012). Seine Basiskategorie des Zeigens erwies sich als ein außerordentlich fruchtbares Instrument für die empirische Analyse von pädagogischen Situationen (Reh et al. 2013). Jemand zeigt jemandem etwas. Damit sind die drei Komponenten des klassischen didaktischen Dreiecks in eine ganz spezifische Beziehung zueinander gesetzt, die den Kern des Pädagogischen auf den Punkt bringt. Das Zeigen hat dabei sowohl eine soziale als auch eine thematische Seite: Es wendet sich an einen anderen, das ist die soziale Seite dieser Geste, und es hat eine themen- und sacherschließende Bedeutung (vgl. Prange 2005: 72).

Dabei muss seine soziale Seite nicht notwendig in einer sozialen Situation verwirklicht werden. Prange deutet dies mit dem Beispiel des Autors an, der dem Leser ,etwas“ zeigen und sich dabei selbst im Schatten halten will (ebd.: 79). Hier klingt die Vorstellung einer begrifflichen Grundstruktur an, die unabhängig von ihrer konkreten sozialen Verwirklichung gedacht werden kann. Der Präzisierung einer derartigen Grundstruktur, unabhängig von den möglichen (sozialen oder rein medialen) Erscheinungsformen, widmet sich Sünkels systematischer Entwurf (Sünkel 2002), der weiter unten noch näher vorgestellt wird.

Bislang wurde in der von Prange inspirierten Unterrichtsforschung ausschließlich das Ausüben des Zeigens in sozialen Situationen ins Zentrum gerückt, indem das Zeigen als soziale Praktik im Sinne Schatzkis konzeptioniert wurde (Reh/Rabenstein/Idel 2011: 214f.; Schatzki 1996, 2002). Akzentuiert wurde dabei insbesondere die soziale Seite des Zeigens, die unter dem Aspekt der subjektkonstituierenden Adressierung der Lernenden durch die Zeigenden reflektiert wurde (Reh/Rabenstein/Idel 2011). In Anschluss an Butler (2009) geht es um die Frage, zu wem ein/-e Schül/-er/-in gemacht wird, wenn er auf je spezifische Weise im Unterrichtsgeschehen angesprochen wird. 
Auch die thematische Seite des Zeigens kommt - allerdings in Abwandlung von Pranges Entwurf - unter der leitenden Frage ,,zu welcher Sache ein Gegenstand im Zeigen wird" (Reh/Rabensein 2013: 303) in den Blick. Nachdem die Sache nicht als eine vorgängige, unabhängig von der Kommunikation zwischen Lehrperson und Lernenden gedachte Sinnstruktur angenommen wird (wie dies später noch in Sünkels Begriff der „objektivierten Tätigkeitsdisposition“ anklingen wird; vgl. Sünkel 2002: 68), rückt das didaktische Agieren des Zeigenden, das als Vermittlungshandeln auf die zu zeigende Sache hin orientiert ist, in den Hintergrund.

Wie sich diese beiden unterschiedlichen Konzeptualisierungen der Sache auf die konkrete Interpretationsarbeit auswirkt, lässt sich an dem folgenden Fallbeispiel verdeutlichen (Idel/Rabenstein 2013: 45): Der Erstklässler Fabian bringt ein Körbchen Pilze mit in die Schule. Die Lehrerinnen fordern ihn auf, die Pilze den anderen Schüler/-innen zu zeigen. Fabian wird deshalb seitens der Autor/-innen als ,Zeigender“ klassifiziert und die Pilze dementsprechend als das zu Zeigende, also als die Sache des Unterrichts betrachtet. Der performative Akt des Zeigens wird hier bereits als pädagogische Geste des Zeigens, des Verweisens auf die Sache gedeutet, als die sie aus einer didaktischen Perspektive im engeren Sinne jedoch noch nicht verstanden werden würde. Demnach wären die Pilze zunächst lediglich Dinge, die der Junge mitgebracht hat und nun vorzeigen soll. Zum Unterrichtsgegenstand werden sie erst ab dem Zeitpunkt, als die Lehrerin beginnt, Fragen zu stellen, und die Pilze (als Gattung) thematisiert. Erst durch dieses didaktische Agieren wird sie zu einer Zeigenden (im Sinne Pranges), die die Aufmerksamkeit der Schüler/-innen (mittels ihrer Fragen) auf die Gattung der Pilze und ihrer Eigenschaften lenkt. Die Pilze avancieren dadurch vom Unterrichtsaccessoire zum Unterrichtsmedium. Fabian selbst zeigt also nun an dieser Stelle zwar das Unterrichtsmedium vor, ein Zeigender im Sinne Pranges ist er dadurch jedoch noch nicht. ${ }^{3}$ An diesem Beispiel wird deutlich, dass aus einer genuin didaktischen Perspektive nicht alle sozialen Praktiken des Zeigens als pädagogische Grundoperation des Zeigens im Sinne Pranges verstanden werden, ebenso wie diese umgekehrt nicht unbedingt in Form einer ostentativen Geste des Zeigens in Erscheinung treten muss (Prange/Strobel-Eisele 2006). Zu einer pädagogischen Geste des Zeigens werden die unterschiedlichen Praktiken dort erst dann, wenn sie nicht nur beliebig mit einem Objekt in Zusammenhang stehen, sondern eben auf einen Sachverhalt verweisen, der der adressierten Person in seiner Bedeutung erschlossen werden soll (vgl. Prange 2005: 70, 74).

Gegenüber einer derartigen Analyse didaktischen Handelns eröffnet die veränderte Perspektive der Lernkulturanalyse andere, aber ebenfalls bedeutsame Aspekte der Auseinandersetzung mit der Inhaltsdimension des Unterrichts, der als eine ,triadische (gegenstandsbezogene) Kommunikation“ (Tomasello 2011: 40) zwischen Menschen verstanden wird. Zur Erschließung wurde seitens der Lernkulturanalyse eine Heuristik entwickelt, in der die Analyse der Konstitution der Sache im Rahmen unterrichtlicher Kommunikation mit der intersubjektiven Adressierung der Akteure des Unterrichts

3 Der von den Lehrerinnen initiierte performative Akt, den Fabian ausführt, lässt ihn allerdings als Zeigenden (auch im Sinne Pranges) erscheinen. Dass hier - wie die Autoren herausarbeiten - nicht die Expertise des Zeigenden, sondern lediglich seine Inszenierung als Experte im Vordergrund steht (ebd.: 52), wird auch aus didaktischer Perspektive unterstrichen. 
wechselseitig operationalisiert wurde (vgl. Reh et al. 2015: 310ff.). Der Verzicht auf eine vorgängig gedachte Sache des Unterrichts hat allerdings zur Folge, dass die Praktiken der Akteur/-innen nicht als gerichtete Praktiken der Vermittlung und darauf bezogener Aneignung in den Blick kommen und wesentliche Kategorien der Didaktik außen vor bleiben. Damit rückt die Frage nach dem Unterrichtsgegenstand nochmals in den Blick. Um diesen zentralen Begriff noch präziser fassen zu können, als das bislang geschehen ist, soll der bereits oben angesprochene systematische Entwurf Sünkels (2002) einbezogen werden.

Auch Sünkel zielt darauf ab, eine pädagogische Grundstruktur in ihrer elementarsten Form herauszustellen - in diesem Falle allerdings nicht im Blick auf das Phänomen der Erziehung (einschließlich des Unterrichts) wie bei Prange, sondern auf das des Unterrichts (in Abgrenzung zur Erziehung; vgl. ebd.:68). Trotzdem weisen beide Entwürfe große Ähnlichkeit auf. Beide Autoren stoßen aufgrund ihrer systematisch angelegten Reflexionen auf die Form des didaktischen Dreiecks und stellen fest, dass keine vierte Komponente nötig ist, um das anvisierte Phänomen allgemeingültig zu beschreiben (vgl. Prange 2005: 50; Sünkel 2002: 63). Wolfgang Sünkel versteht dabei ganz explizit den Lehrenden und den Schüler als akteurunabhängige Positionen innerhalb der Fundamentalstruktur Unterricht (vgl. Sünkel 2002:65) und damit als unabhängig von ihrer jeweiligen sozialen Rolle als Lehrperson oder als Schüler. Während es bei Prange die Operation des Zeigens ist, die die entscheidende Beziehung zwischen den drei Polen des Dreiecks herstellt, differenziert Sünkel die Beziehungen zwischen den drei Positionen stärker aus. Statt vom Zeigen spricht er allgemeiner von Handlung und Interesse des Lehrenden, aber auch das des Schülers: Während die Aktionsrichtung des Schülers auf den Unterrichtsgegenstand weist, ist das Agieren der Lehrperson eines, das auf eben diese Aktionsrichtung des Schülers hin zur Sache abzielt (vgl. Abb. 2) Es ist also ein „handlungsbezogenes Handeln“ (ebd.:102).

Abbildung 2: $\quad$ Skizze der Fundamentalstruktur des Unterrichts nach Sünkel (vgl. 2002:64)
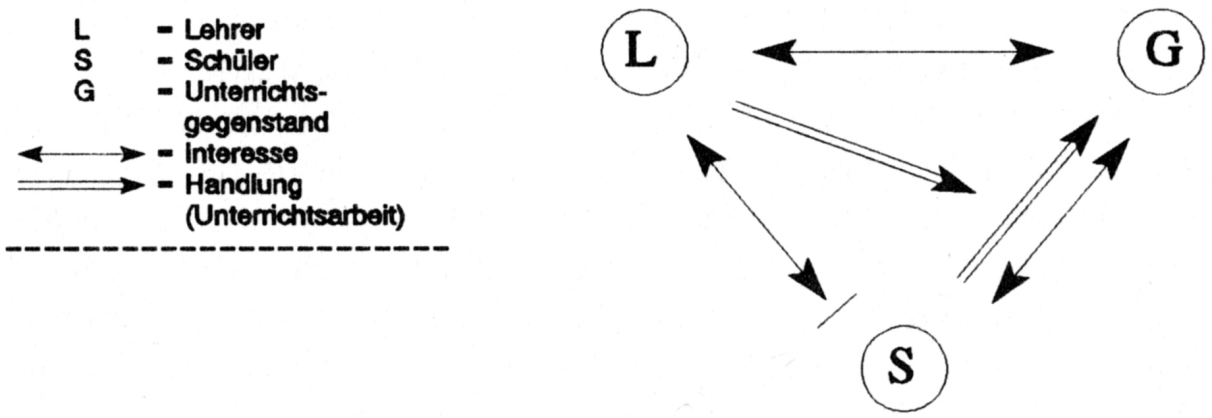

Anders als Prange, widmet sich Sünkel sehr intensiv der Klärung, was ein Unterrichtsgegenstand sei. Denn ,was Unterricht ist, kann nur beantworten, wer auch weiß, was ein Unterrichtsgegenstand ist“" (ebd.:66). Am Beispiel des Bären erläutert er (ähnlich wie Glöckel): 
Der Bär ist offensichtlich ein Gegenstand, und ebenso offensichtlich kann er im Unterricht vorkommen: Unterricht über den Bären ist denkbar. Letzteres ist aber eine theoretisch höchst ungenaue und unsaubere, wenngleich pragmatisch ausreichende, Redeweise, denn der Bär selber kommt in solchem Unterricht nicht in der situativen Position des Unterrichtsgegenstandes vor. Unterrichtsgegenstand ist immer dasjenige, was sich der Schüler im Unterricht aneignet, und das ist nicht der Bär; den Bären kann man essen, nicht lernen. Lernen kann man das Wissen über den Bären. (ebd.:66)

Dass es ein „Wissen über den Bären“ gibt, ist überhaupt die Voraussetzung dafür, dass etwas systematisch und planvoll vermittelt werden kann, oder allgemeiner gesprochen, dass das Phänomen Unterricht überhaupt entstehen kann. Dieses „Wissen über“ fasst Sünkel mit dem Begriff der objektivierten Tätigkeitsdisposition und stellt fest:

Mögliche Unterrichtsgegenstände sind alle objektivierten Tätigkeitsdispositionen. Alle Gegenstände, die dies nicht sind, also auch alle Tätigkeitsdispositionen, die nicht objektiviert oder nicht objektivierbar sind, können keine Unterrichtsgegenstände sein. (Sünkel 2002: 68)

Ausgehend von diesen Überlegungen zum Unterrichtsgegenstand entwickelt Sünkel seine Definition von Unterricht: „Wo immer objektivierte Tätigkeitsdispositionen vermittelt und angeeignet werden, handelt es sich um Unterricht" (ebd.:68). Aus seiner Sicht liegt hier auch ,das fundamentale Merkmal, durch welches sich der Unterricht von allen anderen pädagogischen Phänomenen unterscheidet“" (ebd.), z.B. von Erziehung.

Nun bedarf allerdings der Unterrichtsgegenstand, bzw. das zu Zeigende (im Sinne Pranges) - da sind sich beide einig - der Artikulation, damit das zu Vermittelnde auch von den Adressaten angeeignet werden kann (vgl. Prange 2005: 73f.; Sünkel 2002: 7277). Mit Artikulation ist hier eine Strukturierung des zu Zeigenden gemeint, und zwar in einer doppelten Hinsicht: Das zu Zeigende muss einerseits in einzelne Aufgaben und Problemstrukturen zerlegt werden, die andererseits zeitlich in eine sinnvolle Abfolge gebracht werden müssen. Sünkel nennt diese beiden Aspekte „Sequentierung“ und „Problematisierung“ (Sünkel 2002: 72-77). Das Prinzip, nach dem der Unterrichtsgegenstand artikuliert wird, orientiert sich an der Aneignungsarbeit des Schülers/der Schülerin (vgl. ebd.:76). Anders als Prange, der in der Artikulation „die Brücke zwischen Zeigen und Lernen“ sieht (Prange 2005: 109), betrachtet Sünkel die Aneignungsarbeit des Schülers allerdings als eigenständige und unabhängige Leistung, die jedoch ohne die Vermittlungsarbeit, die Artikulation, des Lehrers nicht auskommt, weil diese ihn seinerseits zur Artikulation seiner eigenen Aneignungsarbeit herausfordert (ausführlicher: Sünkel 2002: 81-86). Sünkel vertritt also weder eine technologische noch eine konstruktivistische Sichtweise und nimmt damit gewissermaßen eine vermittelnde Position zwischen jenen Polen ein, die pointiert als „Lehr-Lern-Kurzschluss“ (Holzkamp 1987) auf der einen und „Lern-Lern-Kurzschluss“ auf der anderen Seite karikiert worden sind (Rabenstein/Idel/Rehm 2013: 3).

Die empirische Annäherung zu Beginn dieses Beitrags und die sich daran anschließende systematische Reflexion in Rückgriff auf Glöckel, Prange und Sünkel legen nahe, den Unterrichtsgegenstand als den zentralen Bezugspunkt für die empirische Analyse zu betrachten, von dem aus erst die Geste des Zeigens bzw. die Vermittlungsarbeit des Lehrenden erschlossen werden kann. Auch die Aneignungsarbeit der Schüler/-innen kann erst von diesem Zentrum aus als eine solche nachvollzogen werden. Beides, sowohl die 
Vermittlungsarbeit der Lehrenden als auch die Aneignungsarbeit des Lernenden weisen damit eine teleologische Struktur auf, der eine sinnverstehende Rekonstruktion Rechnung tragen müsste.

Ähnlich wie man aus einem Bild dessen Perspektivität rekonstruieren kann und zum besseren Verständnis die dazugehörigen Fluchtpunkte lokalisiert, so lässt sich der anvisierte Unterrichtsgegenstand aus den jeweiligen Lehr-Lern-Situationen rekonstruieren - unabhängig davon, ob die Lehrperson den Unterrichtsgegenstand explizit nennt, er implizit bleibt oder er gar implizit den explizit genannten unterläuft. Manche Aktion oder Gesprächsführung, die ohne dieses Zentrum befremdlich anmuten (vgl. Gruschka 2013: 20f.), würden von dort her verständlich werden und einen anderen, unterrichtsspezifischen Sinn bekommen. Das heißt nicht, dass das Befremden über typische unterrichtliche Situationen oder Umgangsformen, das sich mittels sozialwissenschaftlicher Ansätze, wie z.B. der ethnografischen Unterrichtsforschung, einstellt, wertlos wäre. Im Gegenteil, es kann sehr aufschlussreich sein und die Augen für manchen blinden Fleck öffnen. Es allerdings dabei zu belassen, ohne jene Grundstruktur in den Blick zu nehmen, die sich in der sozialen Situation des institutionalisierten Lehrens und Lernens auf je eigene Weise realisiert, würde wichtige Erkenntnismöglichkeiten verschenken.

\section{Das Zeigen der Sache am empirischen Beispiel}

Die bisher angestellten theoretischen Überlegungen sollen im Folgenden am eingangs thematisierten Beispiel aus dem Mathematikunterricht (vgl. Abb.1) veranschaulicht werden. Sie führen zu einer didaktischen Unterrichtsforschung ${ }^{4}$ (Baltruschat 2017 in Vorb.), die - ausgehend vom Unterrichtsgegenstand - das auf diesen bezogene Vermittlungshandeln der Lehrperson und das Aneignungshandeln der Schüler/-innen in den Blick nimmt. Nachdem sich im schulisch-institutionalisierten Unterricht Vermittlung und Aneignung entweder in sozialen Situationen vollzieht oder zumindest von solchen gerahmt wird, kommen nicht nur die akteurunabhängigen Positionen des Lehrenden und des Lernenden in den Blick, sondern auch die Akteur/-innen selbst als Personen, die im Rahmen ihrer institutionellen Rollen als Lehrpersonen und Schüler/-innen miteinander in Beziehung treten, und dies sowohl über ihr Vermittlungs- und Aneignungshandeln als auch in ihrer institutionell gerahmten Begegnung allgemein. Die didaktische Interpretation des Unterrichtsverlaufs bedarf deshalb der Ergänzung durch sozialwissenschaftliche Begriffe und Konzepte (z.B. des Rollenhandelns oder der Adressierungen). Dabei verlaufen die didaktische und die sozialwissenschaftliche Perspektive nicht einfach additiv nebeneinander her, sondern sind ihrerseits - wie man am folgenden Beispiel noch sehen wird - miteinander verschränkt. Der hier erprobte Ansatz steht also der dritten Strömung qualitativ-empirischer Unterrichtsforschung (vgl. 2.) am nächsten, da er einerseits auf sozialwissenschaftliche Konzepte zurückgreift, andererseits aber auf genuin didaktischen Kategorien fußt.

4 Der Begriff Didaktik wird hier in einem weiten Sinne verstanden, indem er nicht nur auf die Vermittlungsarbeit der Lehrperson abzielt, sondern auch den Aspekt der Aneignung seitens der Schüler/-innen (Mathetik) mit einschließt. 


\section{Transkript der Sequenz}

83 L: jetzt lege ich mal eine folie auf wenn ich sie denn finde, ja hier habe ich sie, so. ich möchte nur mal, (---) jetzt- paar äußerungen von euch dazu hören. (3.0) was ihr hier so seht;

84 S?: «lachend» scheiße» (3.0) ((L versucht den OHP anzuschalten))

85 L: ich krieg das ding wieder nicht an so jetzt haben wirs. so, (5.0)

((L projiziert eine Folie mit dem OHP an die Wand))

86 S?: was ist das denn-

87 S?: keine ahnung;

88 S?: irgendso striche-

89 Ludger: ein parallelogramm.

90 S?: (ha ha)

91 L: äh (---) ruhig melden wer irgendwas- ludger du hast grad was gesagt?

92 Ludger: das ist ein parallelogramm.

93 L: moritz?

94 Moritz: also die ganzen a::s a: entfernungen sind gleich groß. (---) sieht jedenfalls so aus.

95 L: laura?

96 Laura: ich sag ähm- die beiden zwei also das erste a: ist kleiner als die beiden anderen weil die beiden anderen schief sind;

97 L: [mhm] ((ja))

98 Laura: [und] wenn man die gerade stellen würde wären sie länger- [glaub ich;]

99 L: [mhm] ((ja)) marieke?

100 Marieke: (nee)

101 L: moritz?

102 Moritz: ähm die die die wo das a drin steht sind parallel zueinander und die anderen beiden nicht oder- (---) ja;

103 S?: doch sie sind [parallel zueinander]

104 L: [mhm ((ja)) marco?]

105 Moritz: die anderen [beiden-]

106 Marco: [es sind so] andere winkel- wie soll man das sagen- (---) so die gradzahlen sind anders von den ecken-

$107 \mathrm{~L}: \mathrm{mhm},(\mathrm{ja}))$ robin hat sich noch gemeldet?

108 Robin: ja das ganz rechte ist äh länger also das längste- (---)

109 L: mhm, ((ja)) (2.0)

$110 \mathrm{~L}:$ du hast was gesagt malte?

111 Malte: ja.

112 L: scht äh ja, marieke?

113 Marieke: das erste a: ist glaub ich ein rechteck und die zwei anderen a:s sind parallelogramme114 L: mhm äh könntet ihr mal- hier ist noch ne meldung ludger?

115 Ludger: ja das linke a: ist auch schief also das ist auch ein parallelogramm;

116 L: is auch nicht ganz- ist auch nicht ganz_n rechteck rechter winkel is glaub ich nicht, äh könntet ihr mal vermutungen, zu unserem thema der stunde, (2.0) schließen; fläche und umfang; vermutungen aufstellen- einfach zu diesem bild zu dieser darstellung; ich nehm euch einfach wieder nur nacheinander dran ludger-

117 Ludger: äh das rechte hat den größten umfang würd ich sagen- 
118 L: mhm ((ja)) (2.0) moritz-

119 Moritz: das linke hat den kleinsten umfang.

120 L: mhm ((ja))

121 S?: und das in der mitte- «lachen»

122 L: marieke?

123 Marieke: ich würd mal vermuten dass alle dieselbe fläche haben;

124 L: mhm ((ja)) laura?

125 Laura: glaub ich nicht- ich sage ähm zwischen dem ersten und dem zweiten a: ist gibt es ne größere fläche als zwischen dem zweiten und dem dritten. (2.0)

126 L: robin was sagst du dazu?

127 Robin: ja- dass alle die gleiche fläche haben glaub ich auch.

128 L: mhm ((ja)) marco hast du auch ne meinung?

129 Marco: ja das wollt ich auch sagen;

130 L: ach so. gut. gut ich lass die meinungen jetzt einfach mal stehen- vielleicht gucken wir uns am ende der stunde das nochmal an, und sind vielleicht zu einem ganz anderen ergebnis gekommen ich möchte jetzt einfach- dass ihr bitte, in- (2.0) gruppen ...

Betrachtet man diese Sequenz als Gespräch zwischen Lehrerin und Schüler/-innen, ohne der Sinnstruktur der Grafik als Bezugspunkt Aufmerksamkeit zu schenken, wie dies in der gesprächsanalytischen Interpretation gemacht wurde (Kern 2014), so mutet dieses Gespräch tatsächlich recht eigentümlich an und es ist nachvollziehbar, dass diese Art der Gesprächsführung als „Rätselraten über eine Folie“ (ebd.: 117) kritisiert und das „unklare Handlungspotenzial“" seitens der Lehrerin (ebd.: 115f.) beklagt wird (vgl. Baltruschat 2014: 160). Bezieht man, jenseits des gesprochenen Wortes, die performative Dimension mit ein und betrachtet diese Situation beispielsweise aus einer praxistheoretischen Perspektive mit Fokussierung auf die pädagogische Praktik des Zeigens, so würde man zwar das Zeigen der Lehrerin im Blick auf die Folie thematisieren, eine Analyse der Grafik vom Unterrichtsgegenstand her würde man allerdings kaum durchführen, sondern stattdessen die verhandelte Sache aus den Interaktionen zwischen Lehrerin und Schüler/-innen rekonstruieren (vgl. Reh/Rabenstein 2013: 303). Damit bliebe jedoch jener Fluchtpunkt außen vor, von dem her sowohl das Zeigen der Grafik als auch die ,minimalen Rückmeldesignale“ (Kern 2014: 118) der Lehrerin ihre Bedeutung gewinnen.

Analysiert man die Grafik auf der Folie im Blick auf den Unterrichtsgegenstand dieser Sequenz, also im Blick auf den Zusammenhang zwischen Flächeninhalt und Umfang von Parallelogrammen, ${ }^{5}$ so stellt man fest, dass in der Abbildung selbst bereits

5 Kurz bevor die Lehrerin die Folie auflegt, nennt sie explizit das Thema der Stunde: also - ihr merkt schon - thema dieser stunde ist fläche und umfang; (3.0) und jetzt vom parallelogramm wieder. Dabei schreibt sie „Fläche und Umfang v. Parallelogr.“ als Überschrift über die Skizze an die Tafel. In pragmatisch abgekürzter Redeweise (vgl. Sünkel 2002, S. 66 ; s.o.) könnte man den Unterrichtsgegenstand also mit „Fläche und Umfang von Parallelogrammen“ bezeichnen. Durch die „Artikulation“ in der Grafik, welche drei Parallelogramme mit (erschließbar) gleichem Flächeninhalt und deutlich sichtbar unterschiedlichem Umfang zeigt, kommt der Zusammenhang zwischen Flächeninhalt und Umfang in den Blick. Von den Schüler/-innen werden zunächst einzelne Bestimmungsstücke der Parallelogramme thematisiert (Z. 86-115). Nach dem erneuten Hinweis der Lehrerin auf das thema der stunde, nämlich fläche und umfang (Z. 116), vergleichen die Schüler/-innen die Parallelogramme 
eine Artikulation des Unterrichtsgegenstandes enthalten ist, die es ermöglicht, diesen Zusammenhang aus der Art der grafischen Darstellung zu erschließen. Die drei unterschiedlichen Parallelogramme, die sich in der Abbildung identifizieren lassen (vgl. Abb. 1), sind nämlich zwischen zwei Parallelen gesetzt, die oben und unten verlaufen. Nachdem Parallelen sich dadurch auszeichnen, dass sie stets einen gleichbleibenden Abstand zueinander haben, lässt sich aus dieser Art der Abbildung schließen, dass alle drei Parallelogramme dieselbe Höhe haben. Über jeder der drei Grundseiten der Parallelogramme steht derselbe Buchstabe (a). Er könnte als Hinweis darauf aufgefasst werden, dass diese Seiten ebenfalls gleich lang sind. Aus diesen Indikatoren kann (insbesondere vor dem Hintergrund der soeben vorangegangenen Wiederholung an der Tafel) geschlossen werden, dass alle drei Parallelogramme denselben Flächeninhalt haben - und das, obwohl die schräg verlaufenden Seiten ganz offensichtlich unterschiedlich lang sind, die Parallelogramme also im Umfang verschieden sind. Somit ist in der Grafik bereits eine Zeigegeste im Sinne Pranges (2005) enthalten, bzw. es wird darin eine geleistete Vermittlungsarbeit im Sinne Sünkels sichtbar (vgl. Sünkel 2002: 161). Sie geht auf denjenigen zurück, der diese Grafik erstellt hat (möglicherweise die Lehrerin selbst). Die Lehrerin unterstreicht ihrerseits diese Zeigegeste, indem sie die Folie auflegt, dann beiseite tritt und eine Aufgabe formuliert, die die Hinwendung zur Grafik erfordert. ${ }^{6}$

Die Aneignungsarbeit der Schüler/-innen zeigt sich in einer allmählichen Annäherung an den Unterrichtsgegenstand, wobei die Äußerungen der Schüler/-innen auf die der vorhergehenden Mitschüler/-innen aufzubauen scheinen. Man könnte hier von einer spezifischen Form von Kooperation sprechen, die in der ,,polyadischen Interaktion“ (Krummheuer 2007: 65) dieses Klassengesprächs sichtbar wird. Sie entwickelt sich beginnend mit „,keine ahnung“ über „,sind striche“ bis hin zu ,das ist ein parallelogramm“ und ,a ... gleich groß“. Diese Kooperation kommt erst dann in den Blick, wenn man einerseits die Äußerungen der Schüler auf die Grafik (und den Unterrichtsgegenstand) bezieht und andererseits die Beiträge der Schüler/-innen nicht einfach nur dyadisch als Re-Akte auf die Lehrerin (oder die Folie) hin deutet, sondern einen (impliziten) Bezug aufeinander nicht ausschließt. Krummheuer macht auf eine derartige Perspektive durch die Kategorien des „Lauschers“ oder des „Zuhörers“ (Krummheuer 2007: 69) aufmerksam. Diese Bezeichnungen machen deutlich, dass Schüler/-innen sich auch dort (inhaltlich) aufeinander beziehen können, wo sich dies nicht gleich offensichtlich in entsprechenden Sozial- oder Aktionsformen dokumentiert.

Schließlich gibt die Lehrerin den Hinweis, die Grafik in Blick auf das „Thema der Stunde“, also hinsichtlich „Fläche und Umfang“ von Parallelogrammen, zu betrach-

miteinander hinsichtlich ihres Umfangs und ihres Flächeninhalts. Sie thematisieren den gleich bleibenden Flächeninhalt und den unterschiedlichen Umfang (Z. 117-129). Die Lehrerin selbst expliziert diese Zusammenhänge nicht, sie formuliert nur allgemein fläche und umfang, allerdings unter Verweis auf die Grafik. Anschließend bekommen die Schüler Arbeitsblätter mit Parallelogrammen, deren Umfang und Flächeninhalt sie berechnen sollen. Dazu gibt die Lehrerin den Hinweis vielleicht kommt ihr fällt euch irgendwas auf - und verweist damit wiederum nur indirekt auf den Zusammenhang zwischen Flächeninhalt und Umfang. Der Unterrichtsgegenstand bleibt in dieser Sequenz also implizit.

6 Unter Rückgriff auf Sünkel (vgl. 2002: 158f.), könnte man bei der Verdopplung der Zeigegeste von einem homologischen Lehrersystem sprechen, da sich genau genommen die Position des Lehrenden verdoppelt. 
ten. An dieser Stelle vollführt die Lehrerin eine weitere Zeigegeste in Richtung der Zeigegeste in der Grafik. Die Schüler/-innen vergleichen daraufhin den Umfang der Parallelogramme (Z. 117-119) und bringen damit deren Unterschiedlichkeit als Selbstverständlichkeit zum Ausdruck.

In ihren Zeigegesten legt die Lehrerin ein „handlungsbezogenes Handeln“ (Sünkel 2002: 102) an den Tag: Sie initiiert ein Handeln der Schüler/-innen in Bezug auf den Unterrichtsgegenstand, denn die „Äußerungen“, die sie von ihnen hören möchte, setzen eine geistige Aktivität voraus, nämlich die Beschäftigung mit der in der Grafik enthaltenen Sinnstruktur.

Die Unbestimmtheit der Aufgabenstellung (,was ihr hier so seht") und das äußerst sparsame Feedback („,mhm“) kann man unter einem didaktisch-thematischen und einem pädagogisch-sozialen Aspekt betrachten: Dadurch, dass die Lehrerin alle Sinnkonstruktionen stehen lässt, engt sie die Gedankenspiele der Schüler/-innen nicht ein und lässt der Dynamik ihres Entdeckens freien Lauf. Indem sie selbst weitestgehend außen vor bleibt, gibt sie zudem den Schüler/-innen, als Gruppe im Klassenverband, die Möglichkeit, sich eigenständig mit der Grafik auseinanderzusetzen und dabei aufeinander Bezug zu nehmen. Gleichzeitig adressiert sie die Schüler/-innen als kompetent genug, sich auch ohne weitere Hilfestellung mit der Grafik beschäftigen zu können. Durch den Verzicht der Lehrerin auf Kommentare, Lob oder Bewertung der Äußerungen wird niemand herausgehoben oder abgewertet. Sie korrigiert nur an einer Stelle ein sachliches Missverständnis (Z. 116), tut dies aber auf eine Weise, in der sie auf ihre Autorität als wissende Lehrerin verzichtet und sich stattdessen in die Gemeinschaft der Vermutenden (,glaub ich nicht“") einreiht. Diese Selbstkundgabe korrespondiert mit ihrer Adressierung der Schüler/-innen. Schon zu Beginn dieser Sequenz hatte sich die Lehrerin mit eigenen Schwächen bereitwillig zu erkennen gegeben, als sie Mühe hatte, den OHP einzuschalten (Z. 84). Das Lachen der Schüler/-innen in dieser Situation wird von ihr weder kommentiert noch sanktioniert, was ihre Souveränität und Rollensicherheit unterstreicht (vgl. Nentwig-Gesemann 2014: 132), denn sie lässt keinen Zweifel daran, dass sie selbst sowohl fachlich als auch organisatorisch den Überblick hat und sich als Entscheidungsinstanz für den weiteren Verlauf betrachtet (Z. 83, 116, 130).

An den teilweise kontroversen Äußerungen der Schüler/-innen, die ohne Aushandlung eines Wahrheitsanspruchs stehen bleiben, wird deutlich, dass in dieser Phase des Unterrichts die Möglichkeit von Gedankenexperimenten als erwünscht betrachtet wird und sich widersprechende Äußerungen kein Problem darstellen. Dies wird am Ende dieser Sequenz auch noch einmal seitens der Lehrerin unterstrichen: ,, gut ich lass die meinungen jetzt einfach mal stehen- vielleicht gucken wir uns am ende der stunde das nochmal an, und sind vielleicht zu einem ganz anderen ergebnis gekommen“.

Die Lehrerin strebt hier also weder eine eindeutige Lösung an, noch wird diese für einen späteren Zeitpunkt sicher in Aussicht gestellt. Selbst das bisher erreichte „ergebnis“ wird in Frage gestellt, obwohl einige der Schüler/-innen die Sinnstruktur im Blick auf den Unterrichtsgegenstand in Ansätzen schon richtig erfasst hatten und sich diesbezüglich bei den Schüler/-innen eine Art (stillschweigende) Einigung einstellte (Z. 123, 127-129).

Sowohl die Vermittlungsarbeit als auch die Spuren der Aneignungsarbeit konnten nur im Rückbezug auf den Unterrichtsgegenstand identifiziert werden. Aber auch die 
Einschätzung der Adressierungen wurde durch diesen Bezugspunkt berührt. Insofern ist die Vergewisserung des Unterrichtsgegenstands nicht nur für die Rekonstruktion der didaktisch-thematischen Dimension von Bedeutung, sondern auch für die soziale.

\section{Theoretische Modellierungen für die empirische Unterrichtsforschung}

Dass die empirische Forschung nicht ohne vorgängige Begriffe auskommt und dass diese das eigene Wahrnehmen strukturieren, ist keine neue Einsicht (vgl. Kant 1787/1968). Am Beispiel des Interaktionsbegriffs wurde dies zu Beginn dieses Beitrags veranschaulicht. Die systematische Arbeit an geeigneten und präzisen Begriffen muss also der empirischen Arbeit vorausgehen, wenn diese zu gehaltvollen Ergebnissen kommen will. Trotz der langen Tradition der Allgemeinen Didaktik werden die Bemühungen um eine theoretische Modellierung des Unterrichts, die sich als Basis für die empirische Unterrichtsforschung eignet, immer noch als unzureichend wahrgenommen und als Desiderat formuliert (Gruschka/Herzog/Mesetz/Proske/Reh 2011; Hünig/Kabel 2014; Geier/Pollmanns 2016). In diesem Beitrag wurde nicht der Versuch gemacht, eine umfassende Modellierung vorzustellen, sondern es sollte lediglich ein als zentral erachteter Begriff, der Begriff des Unterrichtsgegenstands, präzisiert und prominent gemacht werden. Er könnte geeignet sein, ein Spezifikum des Unterrichts so auf den Punkt zu bringen, dass Unterricht sich damit in seiner realen aktuellen und historischen Vielgestaltigkeit empirisch erfassen ließe. Insbesondere die theoretische Arbeit von Sünkel, in der dieser Begriff systematisch ergänzt wird durch die Positionen der Lehrenden und des Schülers hin zu einer Fundamentalstruktur des Unterrichts, könnte den Blick für dieses Spezifische des Unterrichts schärfen und so zu einer soliden Basis für die empirische Forschung werden. Eben dies war auch eine zentrale Absicht seiner theoretischen Didaktik (vgl. Sünkel 2002: 177).

Hervorzuheben ist dabei nochmals, dass der Begriff des Unterrichts, so wie ihn Sünkel fasst, und der Begriff des Unterrichtsgegenstands, der hier als zentraler Angelpunkt vorgestellt wurde, sich nicht notwendig auf eine soziale Situation beziehen (vgl. 3.). Trotzdem, oder gerade deshalb sind diese Begriffe - umgekehrt - geeignet, eine beliebige soziale Situation als Unterrichtssituation auszuweisen oder aber, im Gegenteil, die Abwesenheit von Unterricht auszumachen. ${ }^{7}$

Der Begriff des Unterrichtsgegenstands ermöglicht es, das Handeln der Akteure als Vermittlungs- oder Aneignungstätigkeit zu verstehen und so - jenseits oder in Ergänzung zu sozialwissenschaftlichen Kategorien und Analysen - zu didaktischen (und mathetischen) Aussagen zu kommen. Gleichzeitig verzichtet dieser Begriff, ebenso wie die von Sünkel entwickelte Fundamentalstruktur des Unterrichts (vgl. Sünkel 2002: 11f.), weitestgehend auf normative Setzungen und kommt gänzlich ohne Bildungsbegriff aus. Insofern unterscheidet sich das hier vorgestellte Vorgehen deutlich von der bildungstheoretischen Strukturanalyse nach Andreas Gruschka (2011, 2013), in der programtisch-normative Konzepte („Bildung“ und „Erziehung“) die Unterrichtsforschung leiten (Gruschka 2011: 131), ohne jedoch ein vorgängiges präzises Begriffsinstrumentarium

7 Dies soll nicht die Frage danach ersetzen oder entwerten, was die Akteure selbst unter Unterricht verstehen (vgl. Breidenstein 2008: 111). 
für die empirische Analyse bereitzustellen, sondern ein solches als Ergebnis der empirischen Forschung zu erhoffen (ebd.: 133). Dagegen wurde in diesem Beitrag mit den hier vorgestellten Begriffen des Unterrichtsgegenstands und des Unterrichts lediglich ein begriffliches Instrumentarium für die empirische Analyse des faktischen Unterrichts vorgestellt - nicht mehr und nicht weniger.

Die Frage, was aus einer bildungstheoretischen Perspektive, die Sache des Unterrichts zu sein habe oder wie das didaktische Handeln der Akteure aus einer derartigen Perspektive zu bewerten sei, bliebe andernorts zu klären.

\section{Autorenangaben}

Dr. Astrid Baltruschat

Friedrich-Alexander-Universität Erlangen-Nürnberg

Institut für Pädagogik

http://www.astrid-baltruschat.de

\section{Literatur}

Arnold, Karl-Heinz (2009): Unterricht als zentrales Konzept der didaktischen Theoriebildung und der Lehr-Lern-Forschung. In: Arnold, Karl-Heinz/Sandfuchs, Uwe/Wiechmann, Jürgen (Hrsg.): Handbuch Unterricht. Bad Heilbrunn: Klinkhardt, S. 15-22.

Baltruschat, Astrid (2014): Variationen eines Falls: Drei Interpretationen vergleichend betrachtet. In: Pieper, Irene/Frei, Peter/Hauenschild, Katrin/Schmidt-Thieme, Babara (Hrsg.): Was der Fall ist. Beiträge zur Fallarbeit in Bildungsforschung, Lehramtsstudium, Beruf und Ausbildung. Wiesbaden: Springer VS, S. 151-165.

Baltruschat, Astrid (2015): Unterricht als videografische Konstruktion. In: Bohnsack, Ralf/ Fritzsche, Bettina/Wagner-Willi, Monika (Hrsg.): Dokumentarische Video- und Filminterpretation. Methodologie und Forschungspraxis. Opladen/Berlin/Toronto: Barbara Budrich, S. 267-292.

Baltruschat, Astrid (2017): Didaktische Unterrichtsforschung. Wiesbaden: Springer VS (in Vorb.). Bonnet, Andreas (2011): Erfahrung, Interaktion, Bildung. In: Meseth, Wolfgang/Proske, Matthias/ Radtke, Frank-Olaf (Hrsg.): Unterrichtstheorien in Forschung und Lehre. Bad Heilbrunn: Klinkhardt, S. 189-208.

Breidenstein, Georg (2008): Schulunterricht als Gegenstand ethnographischer Forschung. In: Hünersdorf, Bettina/Maeder, Christoph/Müller, Burkhard (Hrsg.): Ethnographie und Erziehungswissenschaft. Methodologische Reflexionen und empirische Annäherungen. Weinheim/München: Juventa, S. 107-117.

Butler, Judith (2009): Die Macht der Geschlechternormen und die Grenzen des Menschlichen. Frankfurt/Main: Suhrkamp.

Combe, Arno (2013): Perspektiven der Unterrichtstheorie. Eine Diskussion neuerer theoretischer Konzeptualisierungen von UnterrichtIn: Zeitschrift für interpretative Schul- und Unterrichtsforschung 2, 2, S. 158-173.

Dörpinghaus, Andreas/Nießeler, Andreas (Hrsg.) (2012): Dinge in der Welt der Bildung - Bildung in der Welt der Dinge. Würzburg: Königshaus\&Neumann.

Geier, Thomas/Pollmanns, Marion (Hrsg.)(2016): Was ist Unterricht? Zur Konstitution einer pädagogischen Form. Wiesbaden: Springer VS. 
Glöckel, Hans (2003): Vom Unterricht. Bad Heilbrunn: Klinkhardt.

Gruschka, Andreas (2011): Der empirische Blick auf das Unterrichten als pädagogischer Prozess. In: Meseth, Wolfgang/Proske, Matthias./Radtke, Frank-Olaf (Hrsg.): Unterrichtstheorien in Forschung und Lehre. Bad Heilbrunn: Klinkhardt, S. 130-144.

Gruschka, Andreas (2013): Unterrichten - eine pädagogische Theorie auf empirischer Basis. Opladen/Berlin/Toronto: Barbara Budrich.

Gruschka, Andreas/Herzog, Walter/Meseth, Wolfgang/Proske, Matthias/Reh, Sabine (2011): „In der Frage der Unterrichtstheorien stehen wir doch ziemlich am Anfang“ - ein Streitgespräch. In: Meseth, Wolfgang/Proske, Mathias/Radtke, Frank-Olaf (Hrsg.): Unterrichtstheorien in Forschung und Lehre. Bad Heilbrunn: Klinkhardt, S. 242-262.

Hudson, Brian/Meyer, Meinert A. (2011): Introduction: Finding common ground beyond fragmentation. In: Dies. (Hrsg.): Beyond Fragmentation: Didactics, Learning and Teaching in Europe. Opladen \& Farmington Hills: Barbara Budrich, S. 9-28.

Holzkamp, Klaus (1987): Lernen und Lernwiderstand. Skizzen einer subjektwissenschaftlichen Lerntheorie. In: Forum Kritische Psychologie 20, 2, S. 5-36.

Hünig, Rahel/Kabel, Sascha (2014): Was ist Unterricht? Rezension der gleichnamigen Tagung im September 2013 in Halle. In: Zeitschrift für interpretative Schul- und Unterrichtsforschung. 3, 1, S. 144-148.

Idel, Till-Sebastian/Rabenstein, Kerstin (2013): „Sich als Zeigender zeigen“. Verschiebungen des Zeigens in Gesprächsformaten im individualisierenden Unterricht. In: Zeitschrift für interpretative Schul- und Unterrichtsforschung. 2, 2, S. 38-57.

Kant, Immanuel (1787/1968): Kritik der reinen Vernunft. Werke, Bd. 3. Berlin: Gruyter Verlag.

Kern, Friederike (2014): Die Unterrichtssequenz Parallelogramme I - „Was ist hier der Fall“ aus gesprächsanalytischer Sicht. In: Pieper, Irene/Frei, Peter/Hauenschild, Katrin/SchmidtThieme, Babara (Hrsg.): Was der Fall ist. Beiträge zur Fallarbeit in Bildungsforschung, Lehramtsstudium, Beruf und Ausbildung. Wiesbaden: Springer VS, S. 109-122.

Krummheuer, Götz (2007): Kooperatives Lernen im Mathematikunterricht der Grundschule. In: Rabenstein, K./Reh, S. (Hrsg.): Kooperatives und selbstständiges Arbeiten von Schülern. Wiesbaden: VS-Verlag, S. 61-86.

Lüders, Manfred (2011): Die Sprachspieltheorie des Unterrichts. In: Meseth, Wolfgang/Proske, Mathias/Radtke, Frank-Olaf. (Hrsg.): Unterrichtstheorien in Forschung und Lehre. Bad Heilbrunn: Klinkhardt, S. 175-188.

Mead, George Herbert (1968): Geist, Identität und Gesellschaft. Frankfurt/Main: Suhrkamp.

Meseth, Wolfgang/Proske, Matthias/Radtke, Frank-Olaf (Hrsg.) (2011): Unterrichtstheorien in Forschung und Lehre. Bad Heilbrunn: Klinkhardt.

Nentwig-Gesemann, Iris (2014): Die Unterrichtssequenz Parallelogramme II - Fallrekonstruktion mit der Dokumentarischen Methode. In: Pieper, Irene/Frei, Peter/Hauenschild, Katrin/ Schmidt-Thieme, Babara (Hrsg.): Was der Fall ist. Beiträge zur Fallarbeit in Bildungsforschung, Lehramtsstudium, Beruf und Ausbildung. Wiesbaden: Springer VS, S. 123-138.

Nohl, Arnd-Michael/Wulf, Christoph (2013): Mensch und Ding. Die Materialität pädagogischer Prozesse. In: Mensch und Ding. Die Materialität pädagogischer Prozesse. Zeitschrift für Erziehungswissenschaft Sonderheft 25,)- Wiesbaden: VS-Verlag, S. 1-13.

Pieper, Irene (2014): Was der Fall ist: Beiträge zur Fallarbeit in Bildungsforschung, Lehrerbildung und frühpädagogischen Ausbildungs- und Berufsfeldern. In: Pieper, Irene/Frei, Peter/Hauenschild, Katrin/Schmidt-Thieme, Babara (Hrsg.): Was der Fall ist. Beiträge zur Fallarbeit in Bildungsforschung, Lehramtsstudium, Beruf und Ausbildung. Wiesbaden: Springer VS, S. 9-15.

Prange, Klaus (2005): Die Zeigestruktur der Erziehung. Grundriss der Operativen Pädagogik. Paderborn: Ferdinand Schöningh.

Prange, Klaus/Strobel-Eisele, Gabriele (2006): Die Formen des pädagogischen Handelns. Eine Einführung. Stuttgart: Kohlhammer. 
Rabenstein, Kerstin/Idel, Till-Sebastian/Rehm, Markus (2013): Editorial. In: Zeitschrift für interpretative Schul- und Unterrichtsforschung2, 2, S. 3-8.

Reh, Sabine/Fritzsche, Bettina/Idel, Till-Sebastian/Rabenstein, Kerstin (2013): Lernkulturen. Rekonstruktion pädagogischer Praktiken an Ganztagsschulen. Wiesbaden: VS-Verlag.

Reh, Sabine/Rabenstein, Kerstin (2013): Die soziale Konstitution des Unterrichts in pädagogischen Praktiken und die Potentiale qualitativer Unterrichtsforschung. Rekonstruktionen des Zeigens und Adressierens. In: Zeitschrift für Pädagogik 59, 3, S. 291-307.

Reh, Sabine/Rabenstein, Kerstin/Idel, Till-Sebastian (2011): Unterricht als pädagogische Ordnung. Eine praxistheoretische Perspektive. In: Meseth, Wolfgang/Proske, Matthias/ Radtke, Frank-Olaf (Hrsg.): Unterrichtstheorien in Forschung und Lehre. Bad Heilbrunn: Klinkhardt, S. 209-222.

Reh, Sabine/Ricken, Norbert (2012): Das Konzept der Adressierung. Zur Methodologie einer qualitativ-empirischen Erforschung von Subjektivation. In: Miethe, Ingrid/Müller, HansRüdiger (Hrsg.): Qualitative Bildungsforschung und Bildungstheorie. Opladen \& Farmington Hills: Barbara Budrich, S. 35-56.

Reh, Sabine/Fritzsche, Bettina/Idel, Till-Sebastian/Rabenstein, Kerstin (Hrsg.) (2015): Lernkulturen. Rekonstruktion pädagogischer Praktiken an Ganztagsschulen. Wiesbaden: Springer VS.

Schatzki, Theodore R. (1996): Social Practices. A Wittgensteinian Approach to human Activity and the Social. Cambridge/New York: Cambridge University Press.

Schatzki, Theodore R. (2002): The Site oft he Social. A Philosophical Account oft he Constitution of Social Life and Change. University Park- Pennsylvania State: University Press.

Schmidt-Thieme, Babara (2014): Die Unterrichtssequenz ParallelogrammeIII-Interaktionsmuster, Argumentationsstrukturen und Gegenstandskonstitution. Fallanalytische Blicklichter aus mathematikdidaktischer Perspektive. In: Pieper, Irene/Frei, Peter/Hauenschild, Katrin/ Schmidt-Thieme, Babara (Hrsg.): Was der Fall ist. Beiträge zur Fallarbeit in Bildungsforschung, Lehramtsstudium, Beruf und Ausbildung. Wiesbaden: Springer VS, S. 139-150.

Shirley, Dennis (2008): The Coming of Post-Standardization in Education: What Role for the German Didaktik Tradition? In: Meyer, Meinert A./Prenzel, Manfred/Hellekamps, Stephanie (Hrsg.): Perspektiven der Didaktik. Zeitschrift für Erziehungswissenschaft. Sonderheft 9. Wiesbaden: VS-Verlag, S. 35-45.

Silverman, David (2013): Doing Qualitative Research: A Practical Handbook. Los Angeles: Sage. Sünkel, Wolfgang (2002): Phänomenologie des Unterrichts. Grundriss der theoretischen Didaktik. Weinheim/München: Juventa.

Tomasello, Michael (2011): Die Ursprünge der menschlichen Kommunikation. Frankfurt/Main: Suhrkamp. 